\title{
Coagulation Profiles and Viscoelastic Testing in Multisystem Inflammatory Syndrome
}

\author{
Ashish Ankola ${ }^{1}$, Victoria Bradford ${ }^{1}$, Jane Newburger ${ }^{1}$, Sirisha Emani ${ }^{1}$, Audrey Dionne ${ }^{1}$, \\ Kevin Friedman ${ }^{1}$, Mary Beth Son ${ }^{1}$, Lauren Henderson ${ }^{1}$, Pui Lee ${ }^{1}$, Amy Hellinger ${ }^{1}$, Beth \\ Hawkins $^{1}$, Courtney Ventresco ${ }^{1}$, Paul Esteso ${ }^{1}$, and Christina VanderPluym ${ }^{1}$ \\ ${ }^{1}$ Boston Children's Hospital
}

May 8, 2021

\begin{abstract}
Objective: To characterize viscoelastic testing profiles of children with multisystem inflammatory syndrome in children (MISC). Methods: This single-center retrospective review included 30 patients diagnosed with MIS-C from January 1 to September 1, 2020. Thromboelastography (TEG) with platelet mapping was performed in 19 (63\%) patients and compared to age- and gender- matched controls via Student's t-test and Wilcoxon rank sum test. Pearson's and Spearman correlation were used to assess relationships between TEG parameters and inflammatory markers. Results: Patients with MIS-C had abnormal TEG results compared to controls, including decreased $\mathrm{K}$ time (1.1 vs. $1.7 \mathrm{~min}, \mathrm{P}<0.01)$, increased alpha angle (75.0 vs. 65.7 degrees, $\mathrm{P}<0.01)$, increased maximum amplitude $(70.8$ vs. $58.3 \mathrm{~mm}, \mathrm{P}<0.01)$, and decreased Ly-30 (1.1 vs. 3.7\%, $\mathrm{P}=0.03)$; consistent with increased clot formation rate and strength, and slower fibrinolysis. TEG maximum amplitude was moderately correlated with erythrocyte sedimentation rate $(\mathrm{r}=0.60, \mathrm{P}=0.02)$, initial platelet count $(\mathrm{r}=0.67, \mathrm{P}<0.01)$, and peak platelet count ( $\mathrm{r}=0.51$, $\mathrm{P}=0.03)$. TEG alpha angle was moderately correlated with peak platelet count $(\mathrm{r}=0.54, \mathrm{P}=0.02)$. 17 (57\%) patients received aspirin (ASA) and anticoagulation, $5(17 \%)$ received only ASA, and $3(10 \%)$ received only anticoagulation. No patients had a thrombotic event. $6(20 \%)$ patients had a bleeding event, none of which was major. Conclusions: Patients with MIS-C had evidence of hypercoagulability on TEG. Increased erythrocyte sedimentation rate and platelets were associated with higher clot strength. Treatment with ASA or anticoagulation was well tolerated. Further multi-center study is required to characterize the rate of thrombosis and optimal thromboprophylaxis algorithm in this patient population.
\end{abstract}

\section{Introduction}

Coronavirus disease 2019 (COVID-19), caused by the novel coronavirus SARS-CoV-2, has had a tremendous impact since the first cases were reported in China in December 2019. Since April 2020, cases have emerged of healthy children presenting with a severe, immune-mediated, multi-organ system illness following SARSCoV-2 infection, which has since been termed "multisystem inflammatory syndrome in children" (MIS-C). ${ }^{1-5}$ Patients with MIS-C have evidence of significant abnormalities in the inflammatory cascade and hemostasis, including elevated fibrinogen and D-dimer. ${ }^{6-9}$ Reported rates of thromboembolism in the pediatric MIS-C population range from 3 to $6.5 \% \cdot{ }^{6,10,11}$ Derangements in hemostasis in adults with severe COVID-19 infection are more pronounced and better characterized. In the adult literature, SARS-CoV-2 infection is associated with a prothrombotic state, with viscoelastic testing consistent with early thrombin burst, increased fibrin generation, greater clot strength and reduced fibrinolysis. ${ }^{12-14}$ This has been consistent with evidence that approximately $20-40 \%$ of adults with severe acute COVID-19 infection in intensive care units (ICU's) are affected by thromboembolism despite anticoagulation. ${ }^{15-17}$

Thromboelastography (TEG) is a point of care, whole blood viscoelastic test that is widely established in adults and used to characterize clot formation and lysis kinetics as well as clot strength. ${ }^{18}$ Increased 
in vitro clot strength on TEG is associated with increased risk of thromboembolism in critically ill adult patients. ${ }^{19}$ TEG has shown promise in pediatric care, including guiding management of product resuscitation and thrombosis risk. ${ }^{20-22}$ We sought to describe the coagulation and viscoelastic testing profiles of children with MIS-C at our institution, as well as our approach to thromboprophylaxis in these patients.

\section{Methods}

Population: This retrospective case series included patients who were diagnosed with MIS-C and admitted to Boston Children's Hospital from February 1, 2020 through September 1, 2020. This study was approved by our institutional review board.

Data collection and definitions: The electronic medical record was reviewed to obtain patients' demographic, clinical, and laboratory data. Patients were diagnosed with MIS-C per the Centers for Disease Control and Prevention's case definition: age $<21$ years presenting with fever, laboratory evidence of inflammation, and clinically severe illness requiring hospitalization; with multisystem ([?]2) organ involvement; no alternative plausible diagnosis; and positive for current or recent SARS-CoV-2 infection by reverse transcriptase polymerase chain reaction (RT-PCR), serology or antigen test, or COVID-19 exposure within the past 4 weeks. ${ }^{23}$ All MIS-C cases were adjudicated by rheumatologists and cardiologists.

Demographic data including age and gender were collected. Clinical data were collected including baseline comorbidities, hospital course, and any bleeding or thrombosis events. Bleeding was defined per the International Society on Thrombosis and Haemostasis (ISTH): major bleeding (fatal or in critical area or organ), clinically relevant non-major bleeding (requiring intervention by a medical provider, hospitalization/increased level of care, or further evaluation) or minor bleeding (all other events). ${ }^{24,25}$ Thrombosis was described as any progressive or new thrombosis that is clinically symptomatic or diagnosed on imaging, including deep vein thrombosis, sinus venous thrombosis, stroke and pulmonary embolism. Ventricular dysfunction was defined as a left ventricular ejection fraction $<55 \%$. Coronary artery Z-scores were calculated using the Boston formula: coronary artery dilation was defined as coronary artery Z-score [?]2 but $<2.5$ and coronary artery aneurysms were defined as coronary artery Z-score [?]2.5. ${ }^{26}$

Basic laboratory studies related to bleeding and inflammation were collected, including initial and peak values. TEG with platelet mapping data was performed in an institutional laboratory certified by the Clinical Laboratory Improvement Amendments. TEG variables collected included: reaction time (R time, minutes), which measures latency until clot formation begins; kinetic clot time ( $\mathrm{K}$ time, minutes) and rate of clot formation (alpha angle, degrees), which measure velocity of clot formation; maximum amplitude (MA, mm), which measures maximum clot strength; and percentage of clot lysis 30 minutes after the MA is reached (Ly30), which measures fibrinolysis. A hypercoagulable state is characterized by a lower R time, $\mathrm{K}$ time and Ly-30 and a higher alpha angle or maximum amplitude. Platelet mapping was also performed and collected as percent of inhibition of arachidonic acid (AA) and adenosine diphosphate (ADP). TEG data was compared to the profiles from age- and gender- matched controls, who were patients who had TEG profiles collected prior to cardiac surgery, with no anticoagulation or antiplatelet therapies, and with negative SARS-CoV-2 testing.

The use and dosage of prophylactic and therapeutic anticoagulant and antiplatelet therapies were also collected. Treatment course was based on individual clinician preference, with guidance from our institution's Cardiac Antithrombosis Management Program.

Statistical Analyses: Descriptive statistics are presented using means with standard deviations or medians with interquartile ranges for continuous variables as appropriate, and the distribution of variables was visualized using histograms and tests for heteroscedasticity. Frequencies and proportions were presented for categorical variables. TEG with platelet mapping was compared to controls via Student's t-test and Wilcoxon rank sum test. Pearson's and Spearman correlation were used to assess relationships between TEG parameters and inflammatory markers. Significance was determined as $P<0.05$. Statistical analyses were performed with Stata. 


\section{Results}

Cohort Characteristics: Thirty patients were diagnosed with MIS-C during the study period. Median age was $9.5 \pm 5.1$ years and $14(47 \%)$ were male. Twenty-four $(80 \%)$ of patients had significant comorbidities, with the most common being obesity/overweight $(n=17,56 \%)$, followed by asthma $(n=7,23 \%)$. All patients had either positive SARS-CoV-2 RT-PCR $(n=16,53 \%)$ and/or positive antibodies $(n=22,73 \%), 8(27 \%)$ had both. Eight patients (27\%) had an additional documented COVID-19 exposure. Eleven (37\%) patients required admission to an ICU, with $6(20 \%)$ requiring vasopressor support, $5(17 \%)$ requiring positive pressure ventilation, and $1(3 \%)$ requiring mechanical ventilation. Median ICU length of stay was 6 days (interquartile range [IQR] 3-9). On echocardiogram, 7 (23\%) had coronary artery dilation/aneurysm and 17 (57\%) had ventricular dysfunction (mean ejection fraction 54\%, standard deviation [SD] $9.2 \%$ ).

Laboratory Characteristics: Initial and peak laboratory data are described in Table 1. Markers of inflammation, including ferritin, erythrocyte sedimentation rate (ESR), and C-reactive protein (CRP), were elevated on initial presentation. Peak white blood cell count occurred on median day 0 (IQR 0-12) of illness in those not started on steroids, and on median day 8 (IQR 4-12) of illness in those on steroids. Patients did not have significant anemia and were initially mildly thrombocytopenic with a subsequent increase in platelet count later in illness. Liver and renal function was overall normal. Cardiac biomarkers were notable for a mildly elevated median peak b-type natriuretic peptide and a normal median troponin, although $5(17 \%)$ patients had an elevated troponin over the course of illness.

Coagulation profiles are summarized in Table 1. Coagulation profiles were obtained on a median day 0 (IQR 01) of illness, prior to initiation of antiplatelet and anticoagulation medication in 21/24 (88\%) patients started on those therapies. Three patients had coagulation profiles sent 1-2 days after initiating therapies; aspirin in $3 / 3$ and treatment dose low-molecular weight heparin (LMWH) in $2 / 3$, and had no significant prolongation in their coagulation profiles. Baseline values for activated thromboplastin time (aPTT) and prothrombin time (PT) were slightly prolonged, though international normalized ratio (INR) was within normal limits. Peak values were notable for an elevated mean aPTT and PT, with mean INR slightly increased. D-dimer and fibrinogen were elevated on initial presentation and increased later in illness.

TEG Profiles: Table 2 contains serial TEG data over the course of MIS-C illness with patients' corresponding thromboprophylaxis regimens. The initial TEG profile was obtained on day 4 (IQR $3-8$ ) of illness in 19 (63\%) patients. At time of presentation, patients with MIS-C had abnormal TEG profiles compared with ageand gender- matched controls (Figure 1), including decreased $\mathrm{K}$ time $(1.1 \mathrm{vs} .1 .7 \mathrm{~min}, P<0.01$ ), increased alpha angle ( 75.0 vs. 65.7 degrees, $P<0.01)$, increased maximum amplitude $(70.8$ vs. $58.3 \mathrm{~mm}, P<0.01)$, and decreased Ly-30 (1.1 vs. 3.7\%,P =0.03). These findings are consistent with increased rate of clot formation and clot strength, and slower fibrinolysis (depicted in Figure 2). In correlation analysis (Figure 3), TEG maximum amplitude was directly correlated with ESR $(r=0.60, P=0.02)$, initial platelet count $(r=0.67$, $P<0.01)$, and peak platelet count $(r=0.51, P=0.03)$. TEG alpha angle was directly correlated with peak platelet count $(r=0.54, P=0.02)$. No other inflammatory markers were associated with TEG maximum amplitude or alpha angle, including D-dimer, CRP, procalcitonin, ferritin, and fibrinogen. There was no association between TEG alpha angle nor amplitude with any markers of disease severity, including ejection fraction, coronary dilation/aneurysm, inotropic support, intubation or ICU stay.

Over the course of weeks 2-6 of illness, a second TEG profile was obtained in 13 (43\%) patients, and a third TEG profile was obtained in $6(20 \%)$ patients. By the second TEG, 9/11 (82\%) of the patients on ASA had AA inhibition $>50 \%$ and by the third TEG, 6/6 (100\%) of the patients on ASA had AA inhibition > 50\%. There was a significant difference in AA inhibition in those on ASA versus not on ASA; 59.0 (SD 34.6) vs. 4.1 (SD 5.7), $P<0.01$; but no significant difference in clot strength. There was a progressive increase in ADP inhibition over time, despite patients not being on any medications known to cause ADP inhibition.

Treatment Regimens and Adverse Events: Patients were treated with antiplatelet and anticoagulation regimens as summarized in Table 3, with guidance set forth by our institution's Cardiac Antithrombosis Management Program (algorithm used by this team is described in Supplemental Figure S1). Treatment 
is described for all 30 patients with MIS-C, not solely those who had TEG performed. Twenty-two (73\%) patients received aspirin (ASA), 7 (23\%) received prophylactic LMWH, 11 (37\%) received treatment-dose LMWH, $2(8 \%)$ received unfractionated heparin, and $12(40 \%)$ patients received apixaban. Seventeen $(57 \%)$ patients were on dual therapy at one point during their treatment: $14 / 17(82 \%)$ were on ASA and LMWH $(9$ on treatment-dose LMWH, 5 on prophylactic LMWH) and 3/17 (18\%) were on ASA and apixaban (2 were briefly bridged on a heparin drip to apixaban). Only 5 (17\%) patients received no thromboprophylaxis. In $4 / 5$ of these cases, the patients had short hospitalizations and normal echocardiograms, and thromboprophylaxis was not provided based on individual provider preference. One patient had significant thrombocytopenia precluding thromboprophylaxis.

No thrombotic events were documented in this population during their hospitalization or clinical follow-up at our institution. Six (20\%) patients had a total of 7 bleeding events, of which 2 (7\%) were clinically relevant non-major (including bloody stools, epistaxis, or gum bleeding requiring further evaluation or intervention) and 5 (17\%) were minor (including epistaxis, gum bleeding, or bleeding from LMWH sites or sites of trauma). All of the bleeding events occurred when the patient was on some form of anticoagulation or antiplatelet agent: $6 / 7$ events were on ASA, $3 / 7$ were on apixaban, and 2/7 were on LMWH ( 1 treatment-dose, 1 prophylactic). $4 / 7$ events happened while on dual therapy, and $2 / 4$ of these events resulted in discontinuation of the anticoagulant (apixaban) with continuation of ASA. 2/7 events happened while just on ASA, which was stopped as a result. Only one bleeding event occurred on prophylactic LMWH in a patient with bloody stools and a diagnosis of inflammatory bowel disease, and was therefore unlikely to be exclusively attributed to thromboprophylaxis.

\section{Discussion}

This study describes viscoelastic testing profiles in children with MIS-C and demonstrates the presence of a pro-thrombotic state characterized by increased clot formation rate and strength, and slower fibrinolysis. Increased ESR and platelet count were both associated with higher clot strength. The majority of patients received treatment with ASA or anticoagulation, which was well tolerated with $20 \%$ incidence of minor or clinically relevant non-major bleeding events and with no subsequent development of thrombosis.

Our findings build on prior reports in patients with MIS-C that demonstrated laboratory evidence of significant inflammation and coagulopathy. ${ }^{6-10}$ As in our series, children with MIS-C have been reported to have elevations in multiple biomarkers of inflammation, including ESR, CRP, and ferritin. ${ }^{6-8}$ Prior work has demonstrated that baseline platelet count is significantly lower in patients with MIS-C compared to Kawasaki disease. ${ }^{8}$ Although the MIS-C patients in our cohort had thrombocytopenia early in illness, peak platelet counts later in illness were just above the normal range, consistent with prior literature showing a trend towards higher platelet counts upon recovery from MIS-C. ${ }^{9}$ Mild prolongation of PT and aPTT are rarely reported in the MIS-C literature, ${ }^{7}$ but are much more prevalent in adult patients with COVID-19 infection ${ }^{12,27}$ While prolonged PT and aPTT standardly denote increased bleeding risk, in critically ill adults with COVID-19 pneumonia, they have been shown to be associated with increased incidence of thrombotic complications. ${ }^{16}$ In the MIS-C population, elevations in fibrinogen and D-dimer have been extensively described $;^{6-10}$ however, it is unclear whether these laboratory changes reflect an underlying inflammatory state or are indicative of increased risk for thrombosis. ${ }^{10,28}$

Our finding that patients with MIS-C have TEG profiles consistent with faster clot formation, increased clot strength and slower fibrinolysis is novel and provides a potential unique biomarker to distinguish risk for thrombosis in pediatric patients. The MIS-C TEG profiles described in our study are consistent with multiple prior TEG studies in adult COVID-19 patients, which also demonstrate varying degrees of decreased $\mathrm{K}$ time, higher alpha angle, higher maximum amplitude, and low Ly30. ${ }^{12-14}$ One study in pediatric patients with acute SARS-CoV-2 infection (not meeting criteria for MIS-C) found evidence of increased maximal clot firmness on rotational thromboelastometry, another form of viscoelastic testing. ${ }^{29}$ In adults, abnormal TEG profiles predict risk for thrombosis in COVID-19. Indeed, Wright et al showed that patients with a complete lack of clot lysis at 30 minutes and elevated D dimer had a venous thromboembolism rate of $50 \%$ compared to patients with neither risk factor. ${ }^{13}$ In adult patients with COVID-19 infection, TEG with platelet 
mapping, used according to an algorithm to guide anti-thrombotic therapy, can reduce mortality risk. ${ }^{30}$ Our study was not sufficiently powered to detect thromboembolism, particularly as rates of thromboembolism in the pediatric MIS-C population have been reported at $3-6.5 \%, 6,10,11$ far lower than in adults with severe COVID-19. ${ }^{15-17}$ Future studies should explore the role of abnormal TEG profiles in patients with MIS-C as a surrogate for potential thrombosis risk to help guide thromboprophylaxis management.

Proposed mechanisms of thrombosis in adult patients with acute COVID-19 infection include all three components of Virchow's triad. ${ }^{12,31,32}$ Rates of thromboembolism may be lower in pediatric patients with MIS-C due to more robust anti-coagulant pathways associated with developmental hemostasis and baseline healthier endothelial linings. ${ }^{33}$ Our finding that increased platelet counts (both initial and peak) are associated with increased maximal amplitude is consistent with the fact that clot strength is primarily derived from interactions between fibrin and platelets. ${ }^{34}$ Adult COVID-19 literature supports a potential role for increased platelet count and activation in mediating thrombosis risk, although further research is needed in children. ${ }^{35-37}$ The association between degree of ESR elevation and increased clot strength in patients with MIS-C supports a role for inflammation as a potential driver of thromboembolism risk. In adult patients with COVID-19; increased ESR, increased CRP, and increased D-dimer were all predictive of thromboembolic complications. ${ }^{38}$

Although guidelines for management of thromboprophylaxis in patients with MIS-C have emerged, the recommendations are primarily based on evidence in analogous conditions such as Kawasaki disease and adult COVID-19 infection. ${ }^{39-41}$ Low-dose ASA, which was administered to the majority of patients with MIS-C in our study, could be considered in all patients with MIS-C requiring hospitalization, particularly those with features of Kawasaki disease, coronary artery aneurysm, and thrombocytosis. ${ }^{39,40}$ More than half of the patients with MIS-C in our study were on anticoagulation prophylaxis or treatment, with $57 \%$ on dual platelet and anticoagulation therapy. Current guidelines suggest that prophylactic anticoagulation (as well as mechanical thromboprophylaxis) be considered in patients with higher baseline risk for venous thromboembolism (such as patients $>12$ years old, with altered mobility, obesity, known thrombophilia or history of thrombus, or critical presentation) ${ }^{40,41}$ Some guidelines suggest using D-dimer to risk stratify pediatric patients hospitalized for COVID-19 in order to guide initiation of prophylactic anticoagulation, ${ }^{41}$ although this is controversial due to the non-specific nature of an elevated D-dimer. ${ }^{28,40}$ Our institution's algorithm utilizes D-dimer and additionally incorporates TEG clot strength to guide thromboprophylaxis, as described in Supplemental Figure S1. Per current guidelines recommended by the American College of Rheumatology and International Kawasaki Disease Registry, therapeutic anticoagulation should be initiated in patients with MIS-C with giant coronary artery aneurysm (Z-score $>10$ ), moderate or severe ventricular dysfunction, or concern for thrombosis. ${ }^{39,40}$ The lack of major bleeding complications in our cohort suggests that antiplatelet and anticoagulation therapies in the MIS-C patient population are relatively safe.

Limitations of our study include the single center retrospective design, which limits potential generalizability. The small sample size of patients with MIS-C was underpowered to detect the outcome of thrombosis. Due to variations in clinical practice and management of these patients, there were incomplete data particularly with regard to TEG profiles, and there may have been selection bias regarding which patients had TEG profiles obtained. Moreover, we were unable to draw conclusions about the causal relationship between anticoagulation/antiplatelet regimens and outcomes in our cohort due to our retrospective design, small sample size and variable clinical practice. There was no formal control group for our study. TEG data are not routinely obtained in other hospitalized patient cohorts with similar degrees of inflammation; however, they are often obtained prior to cardiac surgery, the patient group from which we derived matched controls.

In conclusion, patients with MIS-C have evidence of hypercoagulability on TEG, particularly in patients with elevated ESR and platelet counts. This study supports the safety and utility of antiplatelet and anticoagulation medication in the management of patients with MIS-C. Further longitudinal multi-center study is required to determine the impact of these therapies on the rate of thrombosis and outcomes in patients with MIS-C.

Conflict of Interest Disclosure: The authors have no conflicts of interest relevant to this article to disclose. 
Funding/Support : Dr. VanderPluym received funding from the Georgia Claire Bowen (GCB) Foundation IMPACT (Imagine More Possibilities for Advanced Cardiac Therapies) innovation lab.

Role of Funder/Sponsor: The funder/sponsor did not participate in the work.

\section{References}

1. Health Department-Reported Cases of Multisystem Inflammatory Syndrome in Children (MIS-C) in the United States | CDC. Accessed March 1, 2021. https://www.cdc.gov/mis-c/cases/index.html

2. Belhadjer Z, Meot M, Bajolle F, et al. Acute heart failure in multisystem inflammatory syndrome in children (MIS-C) in the context of global SARS-CoV-2 pandemic. Circulation. Published online 2020. doi:10.1161/circulationaha.120.048360

3. Verdoni L, Mazza A, Gervasoni A, et al. An outbreak of severe Kawasaki-like disease at the Italian epicentre of the SARS-CoV-2 epidemic: an observational cohort study. Lancet . Published online 2020. doi:10.1016/S0140-6736(20)31103-X

4. Riphagen S, Gomez X, Gonzalez-Martinez C, Wilkinson N, Theocharis P. Hyperinflammatory shock in children during COVID-19 pandemic.Lancet . 2020;395(10237):1607-1608. doi:10.1016/S01406736(20)31094-1

5. Toubiana J, Poirault C, Corsia A, et al. Kawasaki-like multisystem inflammatory syndrome in children during the covid-19 pandemic in Paris, France: prospective observational study. BMJ . Published online 2020. doi:10.1136/bmj.m2094

6. Feldstein LR, Rose EB, Horwitz SM, et al. Multisystem Inflammatory Syndrome in U.S. Children and Adolescents. N Engl J Med . Published online 2020. doi:10.1056/nejmoa2021680

7. Lee PY, Day-Lewis M, Henderson LA, et al. Distinct clinical and immunological features of SARSCoV-2-induced multisystem inflammatory syndrome in children. J Clin Invest. Published online 2020. doi:10.1172/JCI141113

8. Whittaker E, Bamford A, Kenny J, et al. Clinical Characteristics of 58 Children With a Pediatric Inflammatory Multisystem Syndrome Temporally Associated With SARS-CoV-2. JAMA - J Am Med Assoc . Published online 2020. doi:10.1001/jama.2020.10369

9. Del Borrello G, Giraudo I, Bondone C, et al. SARS-COV-2-associated coagulopathy and thromboembolism prophylaxis in children: A single-center observational study. J Thromb Haemost . Published online 2020. doi:10.1111/jth. 15216

10. Aronoff SC, Hall A, Del Vecchio MT. The Natural History of SARS-Cov-2 Related Multisystem Inflammatory Syndrome in Children (MIS-C): A Systematic Review. J Pediatr Infect Dis Soc . Published online 2020. doi:10.1093/jpids/piaa112

11. Whitworth HB, Sartain SE, Kumar R, et al. Rate of thrombosis in children and adolescents hospitalized with COVID-19 or MIS-C.Blood . Published online 2021. doi:10.1182/blood.2020010218

12. Panigada M, Bottino N, Tagliabue P, et al. Hypercoagulability of COVID-19 patients in Intensive Care Unit. A Report of Thromboelastography Findings and other Parameters of Hemostasis. J Thromb Haemost . 2020;(April):1738-1742. doi:10.1111/jth.14850

13. Wright FL, Vogler TO, Moore EE, et al. Fibrinolysis Shutdown Correlation with Thromboembolic Events in Severe COVID-19 Infection.J Am Coll Surg . 2020;231(2):193-203.e1. doi:10.1016/j.jamcollsurg.2020.05.007

14. Salem N, Atallah B, El Nekidy WS, Sadik ZG, Park WM, Mallat J. Thromboelastography findings in critically ill COVID-19 patients.J Thromb Thrombolysis . Published online 2020. doi:10.1007/s11239-02002300-7 
15. Bilaloglu S, Aphinyanaphongs Y, Jones S, Iturrate E, Hochman J, Berger JS. Thrombosis in Hospitalized Patients with COVID-19 in a New York City Health System. JAMA - J Am Med Assoc. Published online 2020. doi:10.1001/jama.2020.13372

16. Klok FA, Kruip MJHA, van der Meer NJM, et al. Incidence of thrombotic complications in critically ill ICU patients with COVID-19. Thromb Res . Published online 2020. doi:10.1016/j.thromres.2020.04.013

17. Helms J, Tacquard C, Severac F, et al. High risk of thrombosis in patients with severe SARSCoV-2 infection: a multicenter prospective cohort study. Intensive Care Med . Published online 2020. doi:10.1007/s00134-020-06062-x

18. Hartmann J, Mason D, Achneck H. Thromboelastography (TEG) Point-of-Care Diagnostic for Hemostasis Management. Point Care . Published online 2018. doi:10.1097/POC.0000000000000156

19. Harahsheh Y, Duff OC, Ho KM. Thromboelastography predicts thromboembolism in critically ill coagulopathic patients. Crit Care Med . Published online 2019. doi:10.1097/CCM.0000000000003730

20. Russell RT, Maizlin II, Vogel AM. Viscoelastic monitoring in pediatric trauma: a survey of pediatric trauma society members. J Surg Res . Published online 2017. doi:10.1016/j.jss.2017.03.016

21. Emani S, Sleeper LA, Faraoni D, et al. Thromboelastography Is Associated With Surrogates for Bleeding After Pediatric Cardiac Operations. Ann Thorac Surg . Published online 2018. doi:10.1016/j.athoracsur.2018.04.023

22. Gong C, Yu K, Zhang N, Huang J. Predictive value of thromboelastography for postoperative lower extremity deep venous thrombosis in gastric cancer complicated with portal hypertension patients. Clin Exp Hypertens . Published online 2020. doi:10.1080/10641963.2020.1836194

23. Information for Healthcare Providers about Multisystem Inflammatory Syndrome in Children (MIS-C) | CDC. Accessed March 1, 2021. https://www.cdc.gov/mis-c/hcp/

24. Schulman S, Kearon C, Subcommittee on Control of Anticoagulation of the Scientific and Standardization Committee of the International Society on Thrombosis and Haemostasis. Definition of major bleeding in clinical investigations of antihemostatic medicinal products in non-surgical patients. J Thromb Haemost . 2005;3(4):692-694. doi:10.1111/j.1538-7836.2005.01204.x

25. Kaatz S, Ahmad D, Spyropoulos AC, Schulman S. Definition of clinically relevant non-major bleeding in studies of anticoagulants in atrial fibrillation and venous thromboembolic disease in non-surgical patients: Communication from the SSC of the ISTH. J Thromb Haemost . Published online 2015. doi:10.1111/jth.13140

26. McCrindle BW, Rowley AH, Newburger JW, et al. Diagnosis, treatment, and long-term management of Kawasaki disease: A scientific statement for health professionals from the American Heart Association.Circulation . Published online 2017. doi:10.1161/CIR.0000000000000484

27. Tang N, Li D, Wang X, Sun Z. Abnormal coagulation parameters are associated with poor prognosis in patients with novel coronavirus pneumonia. J Thromb Haemost. Published online 2020. doi:10.1111/jth.14768

28. Biss TT, Brandao LR, Kahr WHA, Chan AKC, Williams S. Clinical probability score and D-dimer estimation lack utility in the diagnosis of childhood pulmonary embolism. J Thromb Haemost . Published online 2009. doi:10.1111/j.1538-7836.2009.03572.x

29. Al-Ghafry M, Aygun B, Appiah-Kubi A, et al. Are children with SARS-CoV-2 infection at high risk for thrombosis? Viscoelastic testing and coagulation profiles in a case series of pediatric patients.Pediatr Blood Cancer . Published online 2020. doi:10.1002/pbc.28737

30. Hranjec T, Estreicher M, Rogers B, et al. Integral Use of Thromboelastography With Platelet Mapping to Guide Appropriate Treatment, Avoid Complications, and Improve Survival of Pa- 
tients With Coronavirus Disease 2019-Related Coagulopathy. Crit Care Explor . 2020;2(12):e0287. doi:10.1097/cce.0000000000000287

31. The Lancet Haematology. COVID-19 coagulopathy: an evolving story.Lancet Haematol . Published online 2020. doi:10.1016/S2352-3026(20)30151-4

32. Pons S, Fodil S, Azoulay E, Zafrani L. The vascular endothelium: The cornerstone of organ dysfunction in severe SARS-CoV-2 infection. Crit Care . Published online 2020. doi:10.1186/s13054-020-03062-7

33. Kuhle S, Male C, Mitchell L. Developmental hemostasis: Pro- and anticoagulant systems during childhood. Semin Thromb Hemost . Published online 2003. doi:10.1055/s-2003-42584

34. Lam WA, Chaudhuri O, Crow A, et al. Mechanics and contraction dynamics of single platelets and implications for clot stiffening. Nat Mater . Published online 2011. doi:10.1038/nmat2903

35. Hottz ED, Azevedo-Quintanilha IG, Palhinha L, et al. Platelet activation and platelet-monocyte aggregate formation trigger tissue factor expression in patients with severe COVID-19. Blood. Published online 2020. doi:10.1182/blood.2020007252

36. Allegra A, Innao V, Allegra AG, Musolino C. Coagulopathy and thromboembolic events in patients with SARS-CoV-2 infection: pathogenesis and management strategies. Ann Hematol. Published online 2020. doi:10.1007/s00277-020-04182-4

37. Manne BK, Denorme F, Middleton EA, et al. Platelet gene expression and function in patients with COVID-19. Blood . Published online 2020. doi:10.1182/blood.2020007214

38. Al-Samkari H, Karp Leaf RS, Dzik WH, et al. COVID-19 and coagulation: Bleeding and thrombotic manifestations of SARS-CoV-2 infection. Blood . Published online 2020. doi:10.1182/BLOOD.2020006520

39. Henderson LA, Canna SW, Friedman KG, et al. American College of Rheumatology Clinical Guidance for Multisystem Inflammatory Syndrome in Children Associated With SARS-CoV-2 and Hyperinflammation in Pediatric COVID-19: Version 1. Arthritis Rheumatol . Published online 2020. doi:10.1002/art.41454

40. Elias MD, McCrindle BW, Larios G, et al. Management of Multisystem Inflammatory Syndrome in Children Associated With COVID-19: A Survey From the International Kawasaki Disease Registry. CJC Open . Published online 2020. doi:10.1016/j.cjco.2020.09.004

41. Goldenberg NA, Sochet A, Albisetti M, et al. Consensus-based clinical recommendations and research priorities for anticoagulant thromboprophylaxis in children hospitalized for COVID-19-related illness. $J$ Thromb Haemost . Published online 2020. doi:10.1111/jth.15073

FIGURE 1 Comparison of TEG parameters in patients with MIS-C vs. control patients

Data presented as box and whisker blots of median (interquartile range). Statistical significance was determined via Wilcoxon rank sum test.

FIGURE 2 Sample TEG tracing in a patient with MIS-C vs. a control patient

$R=$ reaction time or latency until clot formation begins, $K=$ kinetic clot time or time until clot reaches a fixed strength, angle $=$ rate of clot formation, maximum amplitude $=$ maximal clot strength, Ly30 $=$ clot lysis in 30 minutes after maximum amplitude is reached.

FIGURE 3 Association of TEG parameters with inflammatory markers

Data presented as scatter plots, with statistical significance determined by Pearson or Spearman correlation.

SUPPLEMENTAL FIGURE S1 MIS-C Antithrombosis Management Guide 
This figure describes the algorithm used by our institution's Cardiac Antithrombosis Management Program. Additional abbreviations include $A C=$ anticoagulation, $U L N=$ upper limits of normal, $U F H=$ unfractionated heparin, ECG = electrocardiogram, VTE = venous thromboembolism, DOAC = direct oral anticoagulant.

\section{Hosted file}

Table 1.pdf available at https://authorea.com/users/412632/articles/521257-coagulationprofiles-and-viscoelastic-testing-in-multisystem-inflammatory-syndrome

\section{Hosted file}

Table 2.pdf available at https://authorea.com/users/412632/articles/521257-coagulationprofiles-and-viscoelastic-testing-in-multisystem-inflammatory-syndrome

\section{Hosted file}

Table 3.pdf available at https://authorea.com/users/412632/articles/521257-coagulationprofiles-and-viscoelastic-testing-in-multisystem-inflammatory-syndrome
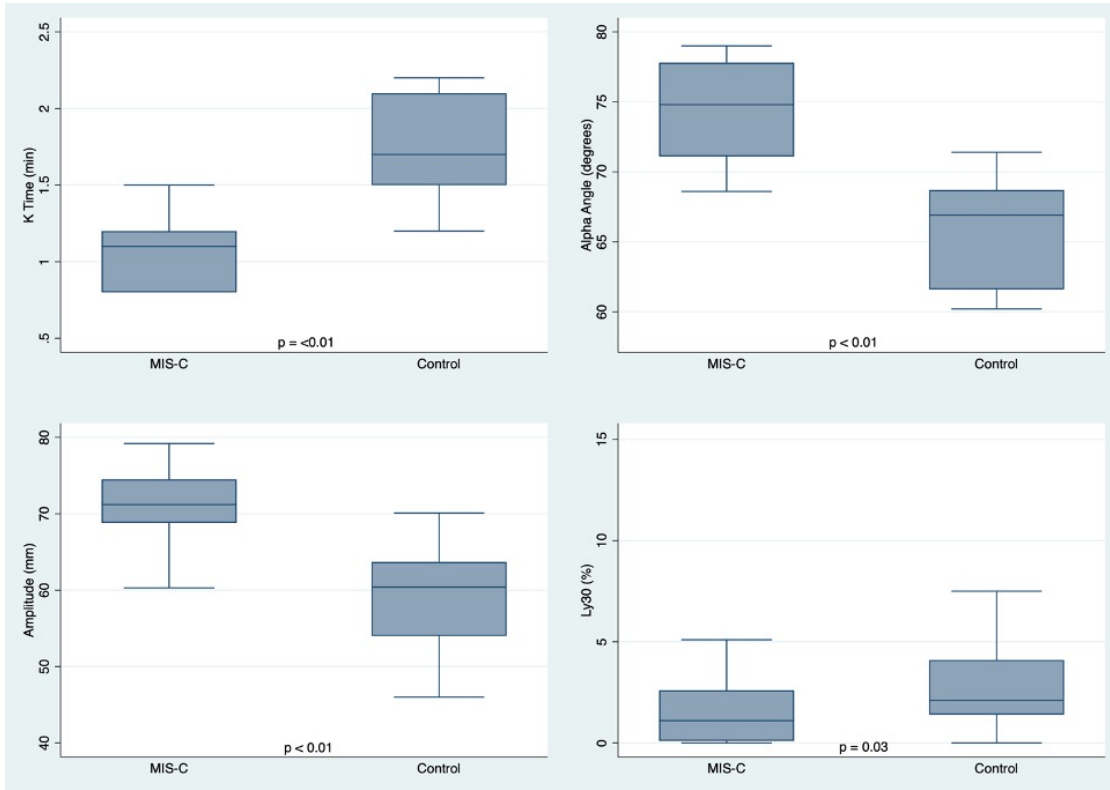

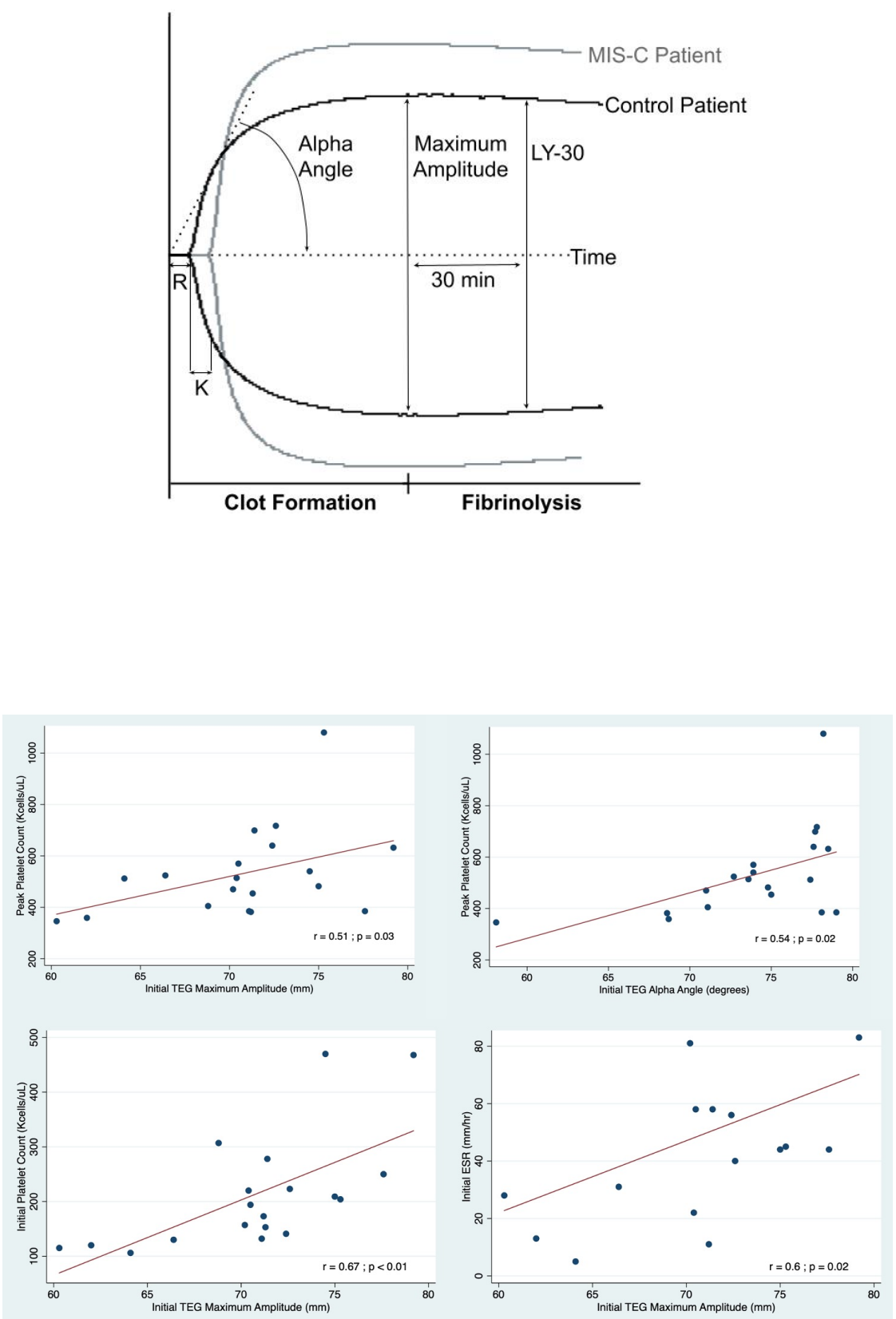


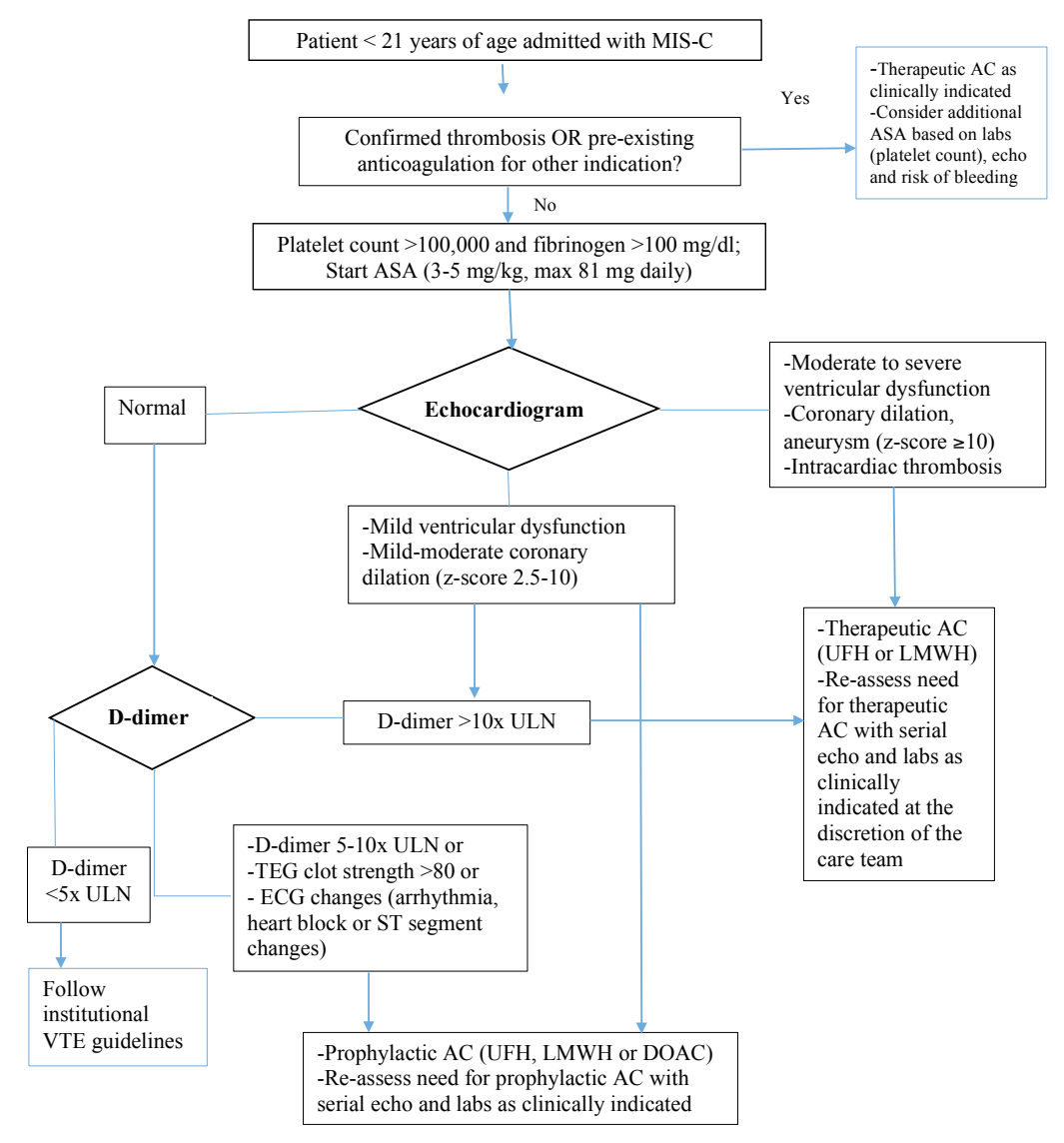

